\section{THE "SCHOTT TREATMENT" OF CARDIAC DISEASE.}

Read in the Section on Tractice of Medicine, at the Forty-eighth Annual Meeting of the American Medical Association at Philadelpbia, Pa. June 1-4, 1897 .

BY C. N. B. CAMAC, M.D.

FIRST ASSIATANT RESIDENT PHYSICIAN IOHNS HOPKINS HOSPITAL, BALTIMORE, MD.

The "Schott treatment" for chronic heart disease, although well established in Germany and England, is so little known in America that it warrants a word of explanation before going on to the description of the cases treated by this method at the Johns Hopkins Hospital.

Nauheim first came to the notice of Europeans in 1834. It was not until 1860 that Dr. Beneke of Marburg considered scientifically the value of the medicated bath treatment. From 1859 to 1870 his articles on the waters of Nauheim made their appearance in the Berliner klinische Wochenschrift; from 1870 to 1890 August and Theodore Schott and J. Groedel were frequent contributors on this subject to this journal. August Schott died, but his brother continued the work and published one of the first articles to appear in English print in 1892. Although this article appeared in the Lancet it received but little attention, and it was not until 1894 that, upon the publication of an article by W. Bezley Thorne, in the Lancet, the treatment came to be discussed in medical circles. Shortly after the appearance of this article, the same author published a systematic explanation of the treatment and how it could be artificially employed. Since the appearance of this book the literature in England and Germany has been crowded with articles on the subject, as may be seen by the extensive bibliography.

Nauheim is situated in the Grand Duchy of Hesse, three-quarters of an hour's ride by train from Frankfurt and two hours' from Homburg, at the eastern slope of the Johannesburg, the last spur of the Taunus mountains. The springs are divided into two classes: those suitable for drinking and those for bathing. The principal ingredients of the waters are sodium chlorid and calcium chlorid, various salts of iron, and an abundance of carbonic acid. Their natural temperature ranges between 82 and 95 degrees F. Spring No. 12 rises some fifty to fifty-six feet above the surface, forming a most beautiful fountain, the waters with their rich supply of carbonic acid gas falling into a seething mass in the basin below.

These waters are led to various houses in which are the bath rooms. Although the waters vary naturally in strength, they are, nevertheless, altered artificially to suit the requirements of the bather. About the central spring are situated these numerous bath houses with their varving strengths indicated by the numbers $1,2,3,4,5$ and 6 . A patient is ordered by his physician to take such a one of these as may be deemed advisable. Learning, therefore, the constituents of these waters and the conditions to which each is applicable, it becomes a mere matter of careful analysis and proper instruction to administer these baths artificially. Fresenius the State chemist at Wiesbaden has given a careful analysis, by following which the artificial baths may be prepared. The baths at the Johns Hopkins Hospital have been conducted upon the basis of these analyses.

It is of interest to inquire first in what way these baths act. That they have been efficacious in the relief of chronic heart disease was made evident before the sub ject was taken up by the brothers Schott. That there is a diminution in the area of cardiac dulness, that there is a change in the position of the apex beat, was also made evident. There was, therefore, under this treatment some distinct change in the degree of congestion of the internal organs and a gradual reduction of a dilated heart. That there was relief from the dyspnea and distress so commonly associated with chronic heart disease, being established on the one hand; that there was a relief of the congestion of the internal organs, evidenced by the reduction of the area of cardiac dulness and an increase in the amount of urine secreted and a diminution of pleural effusions established on the other hand, the question as to how these changes were brought about came to be actively discussed. Throughout the range of literature on this subject, no satisfactory explanation has as yet been given. While Dr. Schott's view, that the salts held in solution pass by imbibition through the outermost layers of the epidermis and so act upon the terminal nerves of the skin, exerting a reflex action on the internal organs, sounds perhaps satisfactory, yet one looks for an explanation which is less of the nature of an hypothesis. In support of his view he mentions that excessive bathing induces an excitable state of the nervous system, sleeplessness and loss of appetite, but this can hardly be considered as proof that the action of the Nauheim waters on the terminal nerves, is account. able for all the changes which result in the internal organs. Nevertheless the conclusion that all these extensive changes are in reality the result of a reflex action produced by influences upon the terminal nerves it must be admitted, is plausible. Thorne's statement, that there is a dilatation of the muscular arteries, and afterward of those of the skin, thus relieving the congestion of the internal organs, is but a part of the explanation and although he adds another link to the chain he by no means completes the explanation. How is this dilatation brought about? True, Dr. Schott states that the calcium chlorid is the active stimulant to the terminal nerves through imbibition. Dr. Lauder Brunton, in his massage experiments, demonstrates that more blood flows through the mas. saged part, and that blood pressure first rises and then falls, but that on the conclusion of massage more blood collects in the massaged parts; experiments which were confirmed by Dr. Oliver.

Ludwig has shown that the capacity of the muscles for blood, is equal to the combined capacities of the internal organs and the skin. In these two experiments, we think we find the most satisfactory explanation of the action of both the baths and the exercises; for, if Dr. Lauder Brunton's experiments in message be correct, and the conclusion of Ludwig be correct, the increased amount of blood in the muscles must indicate a relief of congestion in the internal organs. That there is more blood in the part and necessarily a dilatation of the muscle arteries is proven by increased displacement of the limb. We are yet at a loss to find the link between the action of the salts upon the skin and the increase in the amount of blood in the muscles. If, however, we accept Dr. Schott's explanation of the cutaneous excitation by the gaseous and mineral constituents, followed by a more prolonged stimulation of the sensory nerves produced by a the imbibition of the salts into the superficial layers of the corium, we perhaps, have this missing link in the chain of explanation. To sum up the various theories 
which have been advanced, the calcium chlorid of the salt waters is imbibed into the superficial layer of the corium producing a superficial cutaneous excitation first and then a stimulation of the deeper sensory nerves, these in turn leading to a dilatation of the peripheral vessels with a consequent increase of blood in the muscles and the skin, whose combined capacity far exceeds that of the internal organs, the result being a relief of the congestion of the latter.

Having thus first reviewed the theories in explanation of the way in which these saline waters and the exercises act, the practical questions as to the treatment present themselves. I shall confine myself solely to the administration of the exercises and artificial baths. The course of baths consists of six dif. ferent strengths; the first and second consisting of different proportions of calcium chlorid and sodium chlorid; the third, fourth, fifth and sixth contain carbonic acid as well as these salts. By the expression "course" of six baths is not to be understood that every patient is submitted to the whole six strengths. It simply means that according as the patient improves the bath is increased in strength; for example bath No. 1, may be administered for a period of several days, the patient failing to respond to it; No. 2 , is given and if there be still no response $\mathrm{No}_{0} 3$, and so on even to the full strength, that is bath No. 6. Frequently it is not necessary to go higher than the fourth bath either because the patient shows distinct improvement with the strength already employed or because there is all absence of improvement and his condition contraindicates further submitting him to the treatment.

Bath No. 1, consists of sodium chlorid 4 pounds; calcium chlorid 6 ounces.

Bath No. 2, sodium chlorid 5 pounds; calcium chlorid 8 ounces.

The following baths have together with these salts carbonic acid which is produced by the action of hydrochloric acid and sodium bicarbonate.

Bath No. 3, consists of sodium chlorid 6 pounds; calcium chlorid 10 ounces; bicarbonate soda 6 ounces; hydrochloric acid 7 ounces.

Bath No. 4, sodium chlorid 7 pounds; calcium chlorid 10 ounces; sodium bicarbonate 8 ounces; hydrochloric acid 12 ounces.

Bath No. 5, sodium chlorid 9 pounds; calcium chlorid 11 ounces; sodium bicarbonate 1 pound; hydrochloric acid 1 pound.

Bath No. 6, sodium chlorid 2 pounds; calcium chlorid 12 ounces; sodium bicarbonate 1 pound; hydrochlorid acid 2 pounds.

These various amounts of salt we have put up in packages and labeled bath No. 1, 2, 3, etc., and any strength of bath can be made up by ordering these packages. The calcium chlorid is best prepared in solution. The above proportions correspond to 40 gallons of water, which is just about enough to entirely immerse the body. The bath is given in the morning unless otherwise deemed advisable.

The following are the rules which have been laid down for the administration of the bath. Most of these rules have been made after perusal of the literature and from instructions obtained from Dr. Schott personally.

The bath should be given on an empty stomach; allow the patient to make as little exertion as possible; assist him in every way. A sheet may be drawn over the tub but not around the patient. Be sure that the entire body is immersed. Keep the finger on the pulse during the entire time the patient is in the bath.

Danger signals.-Cyanosis; dyspnea; apnea; inappreciable pulse. On the appearance of any of these, take the patient out of the bath immediately, put him to bed and keep him as quiet as possible. Friction while in the bath is not necessary, but if the fingers and toes become bluish the extremities may be rubbed slightly toward the trunk. Friction should be cautiously employed; when the patient is out of the tub rub him to a glow; give him a glass of milk or cup of bouillon and allow him to rest for an hour.

Diet.-Small quantity q. 4h. meat, boiled chicken, mutton chops; eggs, two a day; oysters raw or panned; vegetables of all kinds; peas, beans, lettuce; liquids; beef tea, bouillon, cocoa, lemonade, milk. NoteNever give more than 4 ounces of fluid at a time. Should be sipped. Wine-Port, Rhine, sherry, brandy; dram to half ounce.

Note.--Something light (cocoa and toast) should be taken one hour before the bath; something light and hot (bouillon, milk punch and toasted crackers) should betaken directly after the bath. If the heart's action is poor, sherry, brandy or port wine may be given after the bath. Last meal to be taken three hours before retiring.

The exercises which are called by Dr. Schott "Widerstandgymnastik" or resistance gymnastics are slow movements executed by the patients and resisted by the physician or operator. The exertion is the very least that can be employed and should cause no fatigue on the part of the patient. Light, loose clothing should be worn by the patient and he should be instructed to breathe quietly. It is hardly necessary to give full explanation of the exercises here as it demands considerable attention and is more in the order of a drill than of an explanation. It is important however to state that these movements should not be administered without thorough training from one competent to give it. The movements are nineteen in number and involve all the larger muscles of the body. This portion of the treatment we have entrusted to our nurses, who have received thorough instruction and whom we find are better adapted to the administration of the light resistance than are men.

Since November we have had under treatment ten cases. Of these ten we have had four deaths, two have shown no improvement, one has shown improvement only during the treatment and three have shown continued improvement.

No one would claim to cure cases of chronic heart disease; all that one can hope for is to place the heart in a functional condition which in relation to the rest of the organism is the best possible compatible with the lesion which exists. In the three cases which have shown continued improvement the results have been very satisfactory, one might say even striking. Of the four deaths No. 1 was a case of aortic insufficiency; No. 2 of dilatation and arterio-sclerosis; No. 3 of chronic nephritis; No. 4 of mitral stenosis. Although the chronic nephritis case was manifestly inappropriate to the treatment, it was selected for the purpose of noting the action upon the kidneys.

In this case, a young girl whose condition at no time was hopeful, the edema of the genitalia and lower extremitities on admission being so extensive that it was impossible to approximate the legs. The features were almost unrecognizable. Beyond a very 
slight response amounting to about 500 c.c., there was no increase in the urine excreted. As an extreme case, therefore, of chronic nephritis this was in no way aided by the treatment. Another case, on the contrary, of chronic interstitial nephritis and tachycardia, the action upon the excretion of urine was most striking. The patient was removed to the isolation ward owing to the unmanageable condition in which she was, following uremia.

The following is a synopsis of the urine record. Record begun December 9.

1. Between December 9 and January 20, forty-three days. Urine fell below 900 c.c., in twenty-four hours, twenty-one times.

Treatment.-Nitroglycerin, continued throughout period of forty-three days; patassium iodid, tr. digitalis, sol. diuretin, sweat baths, cream tartar water, citrate of lithia, apollinaris.

Condition of patient.-Delirious, requiring forcible restraint.

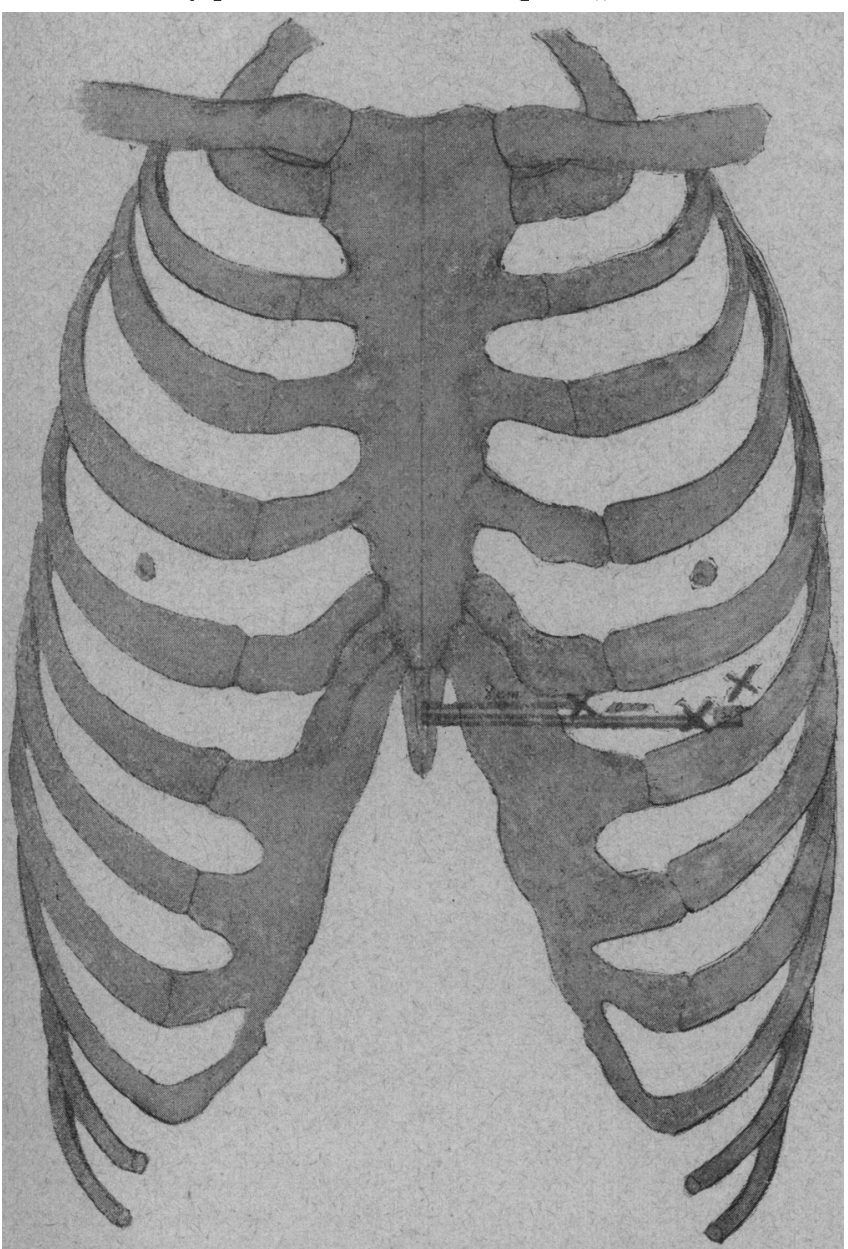

Chart No. 1.-Hunt. Feb. 12. Before bath No. 1, strength No. 1. Point max. imp., 5th space, $181 / 2 \mathrm{~cm}$. from median line; after bath, point max. imp., 5th space, $11 \mathrm{~cm}$. from median line. Feb. 13, no bath given. Point max. imp., 5 th space, $10 \mathrm{~cm}$. from median line. Feb. 14, bath No. 2 strength No. 1. Point max. imp. after bath 5th space, $8 \mathrm{~cm}$. from median line. Feb. 15, no bath given. Point max. imp. not located. No signs (physical or post-mortem) of effusion.

2. Between January 20 and March 2, forty-two days. Urine fell below 900 c.c., in twenty.four hours, four times.

$\square$ Treatment.-Nitroglycerin, apollinaris, Schott baths, hot baths, continued throughout period of forty-two days; tr. strophanthus, tr. digitalis, strychnia, potassium iodid.

Condition of patient.-Rational ; does not sleep well.

3. Between March 2 and 11, eight days. Baths were discon. tinued. Urine fell below 900 c.c., in twenty-four hours, six times.

Treatment.-Tr. digitalis, citrate potash, nitroglycerin, apollinaris, sweat bath, pilocarpin.

Condition of patient.-Dyspnea ; 280 c.c. urine on March 6 210 c.c. urine March 9 ; small irregular pulse.

4. Between March 11 and April 7, twenty-eight days. sisted movements and baths. Urine fell below 900 c.c., in twenty-four hours, five times, upon each occasion with an interval of at least three days.

Treatment.-Nitroglycerin, apollinaris, strychnin, Inf. digit.. Schott bath No. 3, resisted movements Nos. 1, 2, 4, 5, 6, 9 and 10.

Condition of patient.-Out on grounds; sleeps well; no dyspnea. Discharged April 7.

This case we considered as receiving benefit only during treatment. The improvement was not lasting.

The second fatal case (the first being the chronic nephritis in the girl first mentioned) was that of a colored man with marked signs of aortic insufficiency, accompanied by the Flint murmur at the apex. This was an extreme case whose condition had become so serious that he was removed to the isolation ward. So far from the median line was the apex beat that it seemed a suitable case upon which to note the effect of

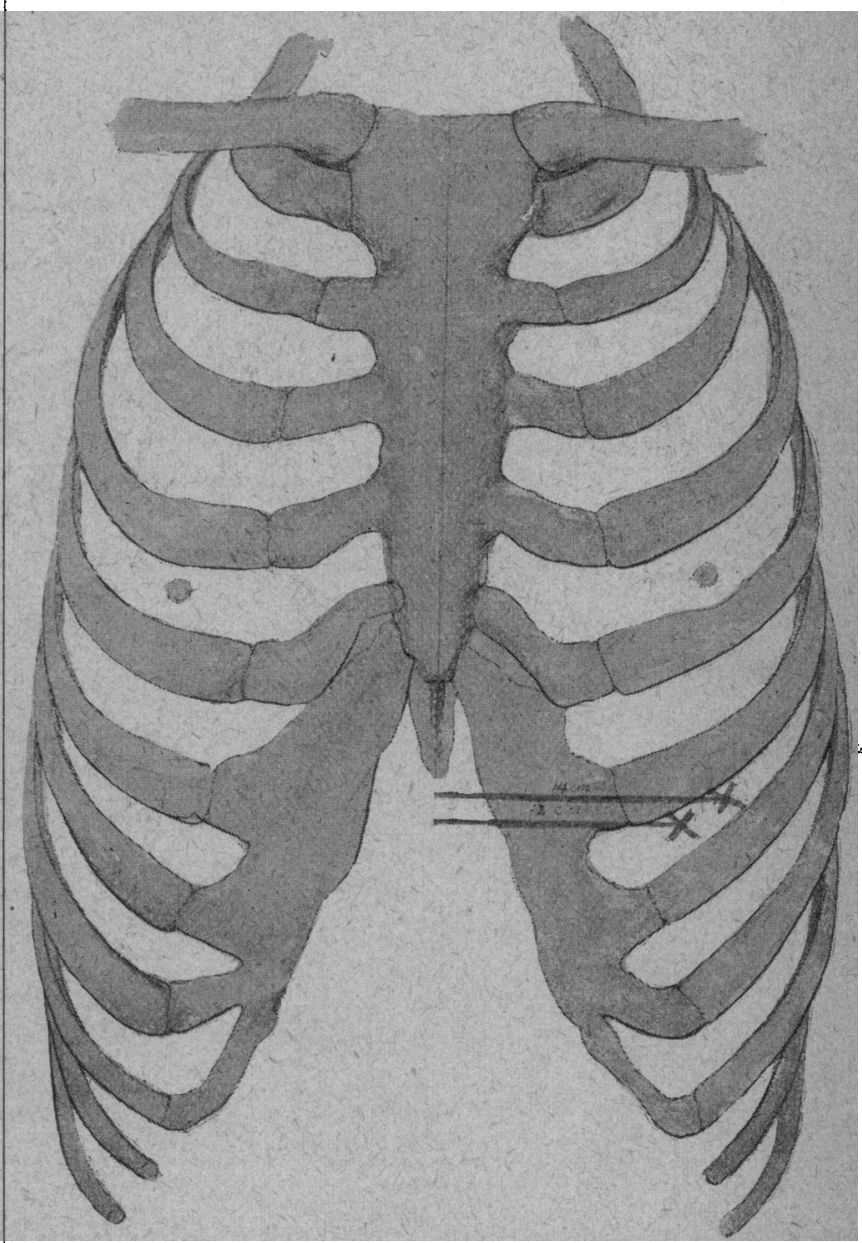

ChaRT No. 2-Larber. Before bath No. 1, strength No.1, point max. mp. $14 \mathrm{~cm}$. from median line. After bath, point max. imp., $12 \mathrm{~cm}$. from median line. Length of time in bath, five minutes.

Charts Nos. 1 and 2 illustrate the immediate influence of the baths upon the point max. imp.

the baths. The point of maximum impulse was seen in the fifth space, $13 \frac{1}{2} \mathrm{~cm}$. from the median line. After the first bath the apex beat was seen in the fifth space $11 \mathrm{~cm}$. from the median line. The day following, the maximum impulse was seen in the fifth space $10 \mathrm{~cm}$. from the median line and on the following day a second bath was given and the apex beat was found in the fifth space $8 \mathrm{~cm}$. from the median line. The patient improved very markedly, became clearer in his head, and was quite manageable. On the 16th, four days after the first bath the patient died suddenly. An effusion at once suggests itself, but there were no signs of such. (Chart No. 1.) 
The third fatal case was a private one who showed the pulse in the case of the cardiac dilatation which signs of cardiac dilatation and a moderate degree of was associated also with arterio-sclerosis; 4 , the ineffithickening of the vessels. This patient was given ciency of the treatment on a case of extreme nephritis. movements from February 21 to April 4. He was The two cases which showed no improvement, extensively edematous, especially the legs. It was one was that of cardiac dilatation, and glycosuria; never possible to make very careful examinations of the other cardiac dilatation with chronic nephritis. the cardiac outline. The pulse, however, which had Although the area of cardiac dulness was diminished been very weak and of small volume. improved very distinctly in the course of the treatment. The edema almost wholly disappeared and the patient from being confined to a chair was able to be up and occasionally to drive. March 29 and 30 , he took a drive and slept well. On March 31 he had a very restless night but in on April 1 was again able to drive. For this patient this was a very great improvement considering he had $w$ and the pulse tracing, which were taken by Dr. never diminished and the patient was discharged practically unimproved (charts $3,4,5$ ). We therefore see that in these seven cases so far reported, those in which the kidneys were extensively involved, no improvement resulted. In those cases in which there

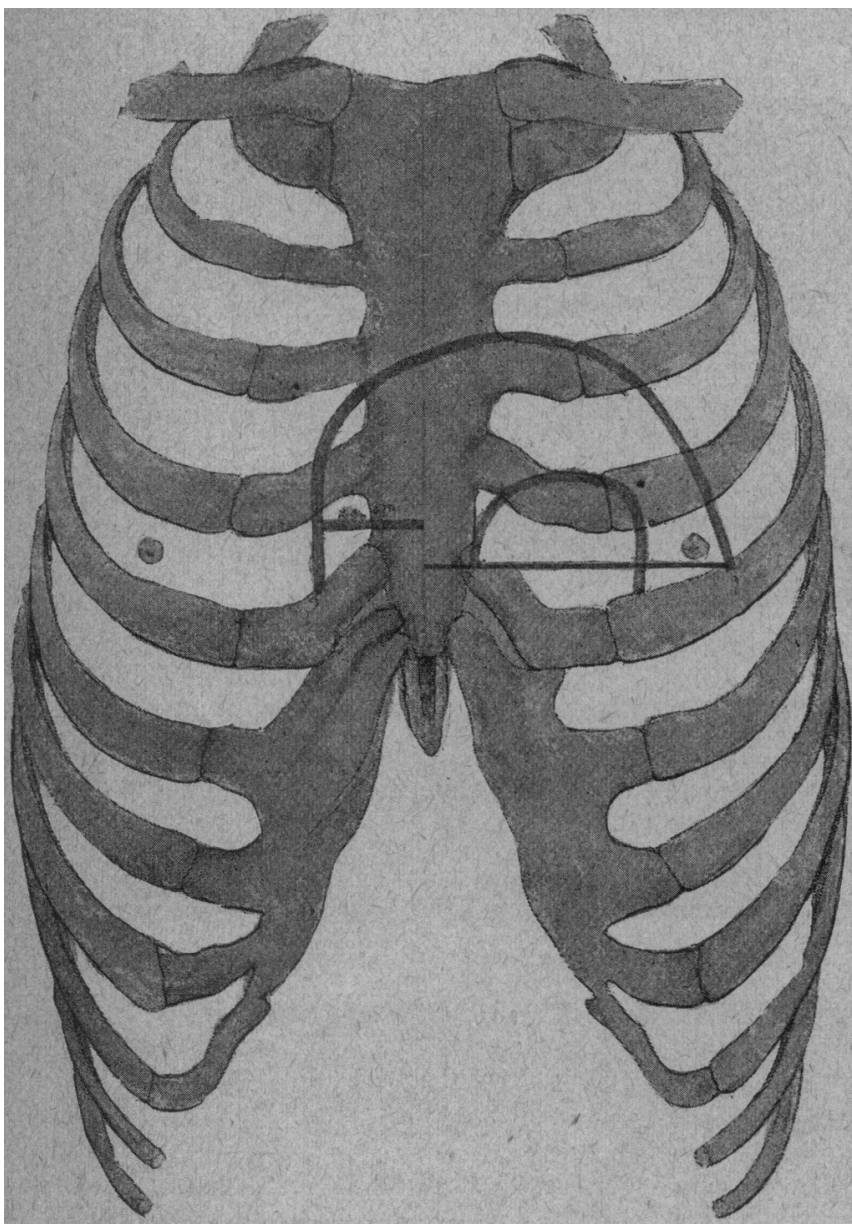

ChaRT No. 3.-Coulbourn, Oct, 13, 1896. Cardiac outline before bath No, 1, strength No. 1. Right border $23 / 4 \mathrm{~cm}$. from median line. In bath eight minutes.

spent months in his room. On April 3 he had a very restless night and from this date he steadily declined, dying suddenly on April 7.

The fourth fatal case was one of mitral stenosis. The change in the apex beat was very striking. This man came in to the hospital suffering from great dyspnea and distress and cyanosis; under the treatment he was able to be up and about the ward, and on the day before Christmas was so much improved as to go to his home. He was, however, an intemperate fellow and got into a spree on Christmas Eve, during which he fell dead. (Chart No. 2.)

In these four fatal cases are shown the marked influence upon, 1 , the position of the apex beat; 2 , the increase in the amount of urine in spite of the nephritis in one case; 3 , the very marked improvement in 


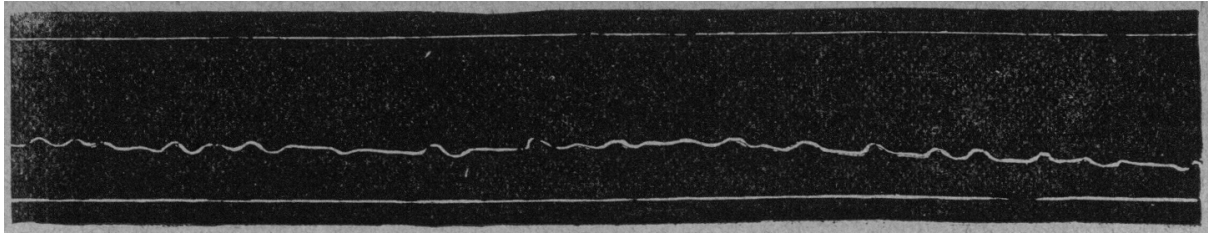

1. October 13, before treatment, $4: 3,4$ hours before first bath.

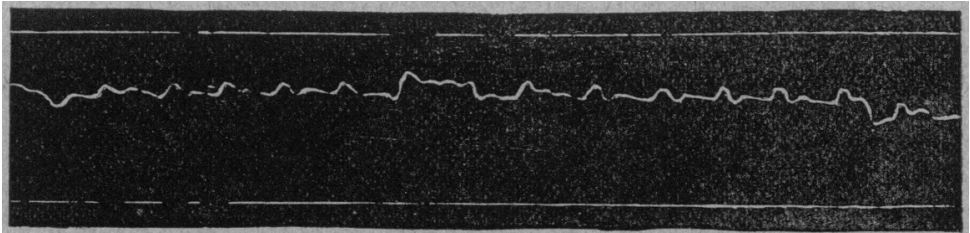

2. October 133. Bath No. 1, strength No, 1 , at $4: 30$ P.M.

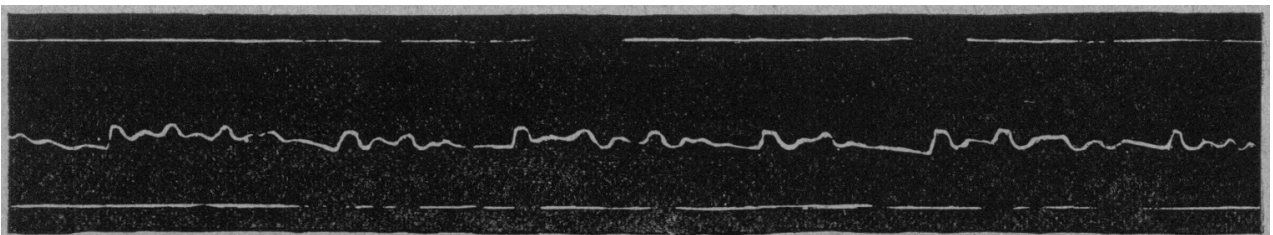

3. ()etober 13, 10 P.M., 5/2 hours after first bath.

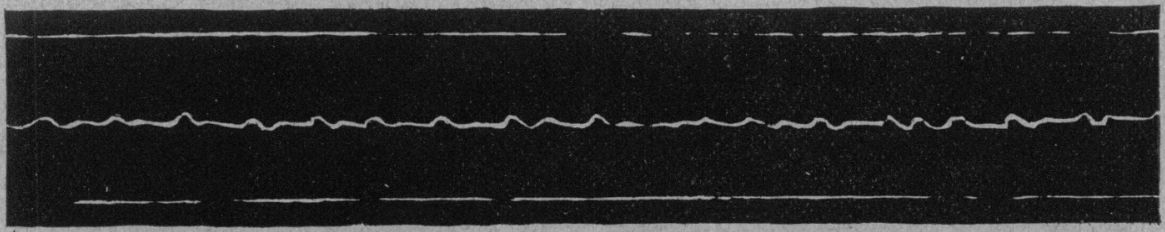

4. Oetober 15, after third bath, strength No. 1.

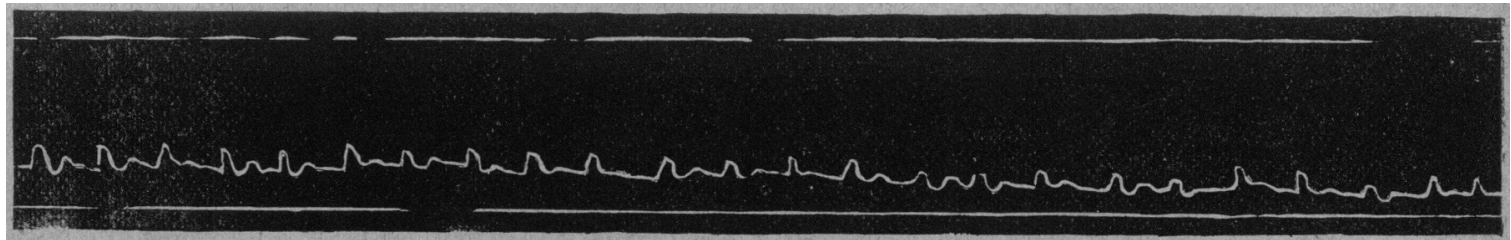

5. Oetober 18 , after sixth bath, strength No. 1 .

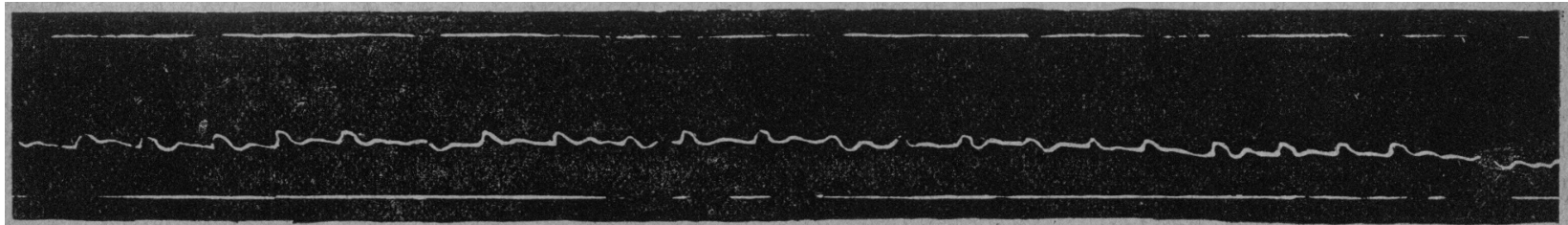

6 October 22 , after eighth bath strength $\mathrm{No}, 2$.

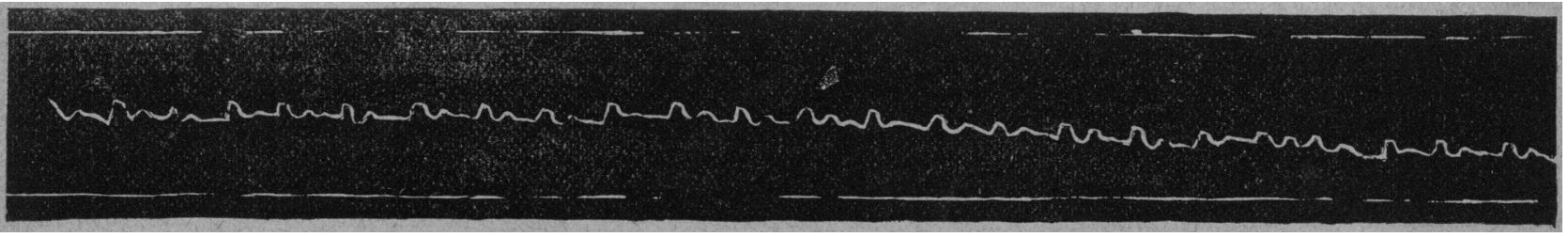

7. Oetober 26 , no batb given on 24 th or 26 th.

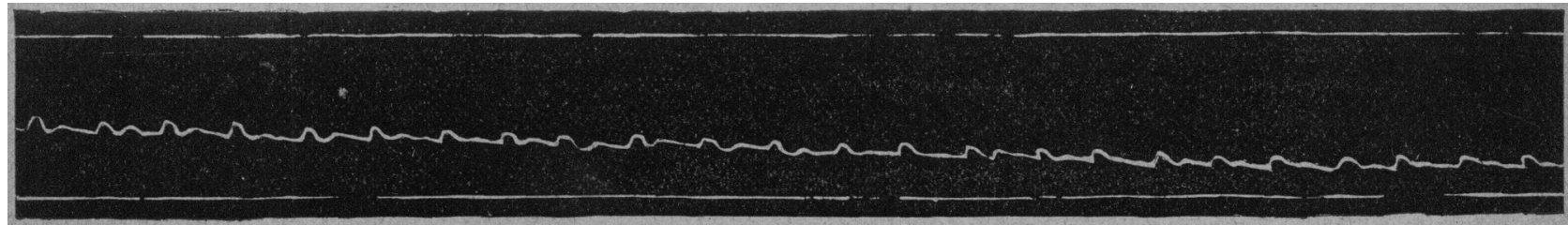

8. October 30 . No baths given since 27 th.

CHART No. i.-Coulbourn. Series of tracings to illustrate the regulating effect of the baths upon the pulse. The dyspnea, however, was so severe that baths had to be discontinued.

Downloaded From: http://jama.jamanetwork.com/ by a DALHOUSIE UNIVERSITY-DAL-11762 User on 05/19/2015 
Schott Method of Baths and Resisted Movements.

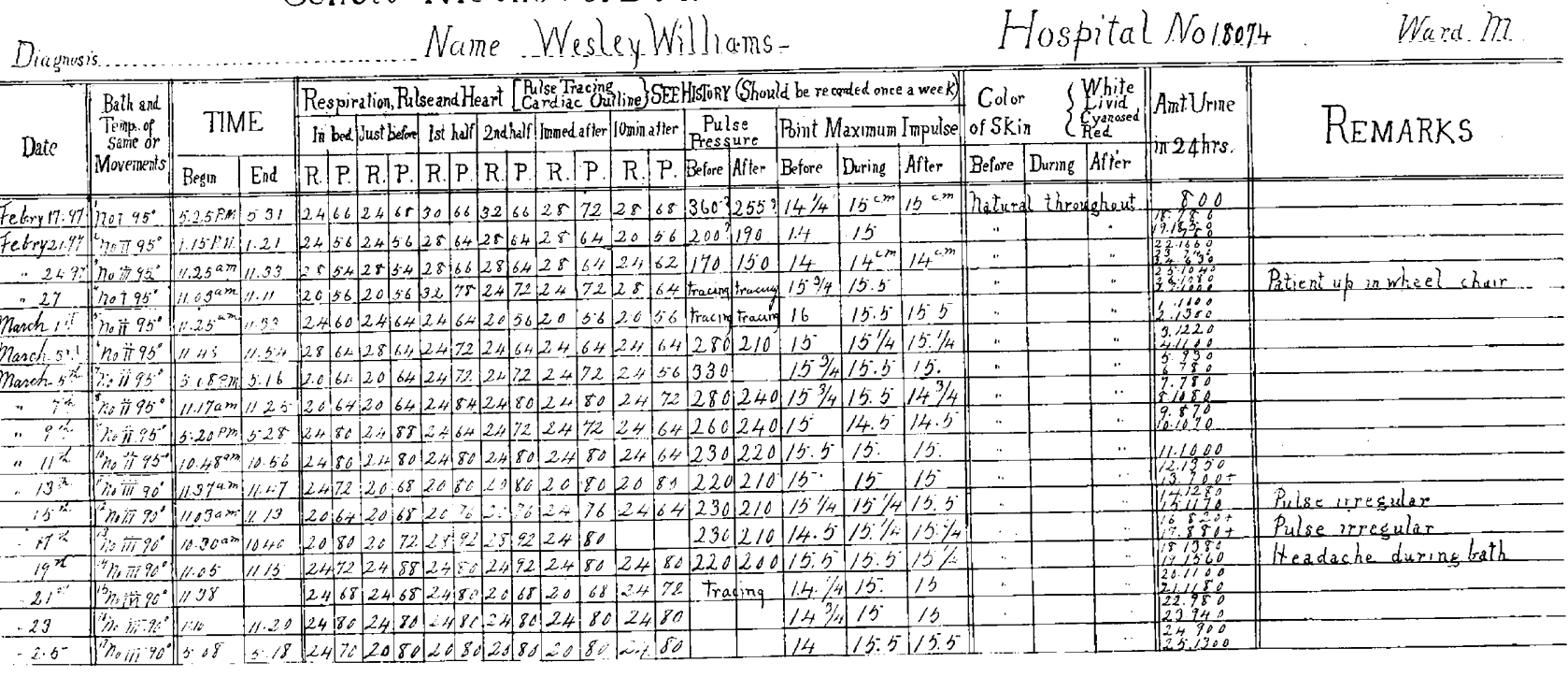

Chart No. 6-Showing method of recording observations. For pulse tracing of this case see Chart No. 7.

while under observation was most comfortable, hav- this case and that of Hauser (Chart No. 8), to be ing no return of his dyspnea or edema. In this case mentioned immediately, with especial reference to the the point of maximum impulse instead of being moved action of digitalis upon the pulse, and that of the in was moved out. (Chart No. 6.)

baths. Chart No. 8 shows the influence of large doses An interesting comparison may be made between of tr. digitalis given for a limited time at short intervals.

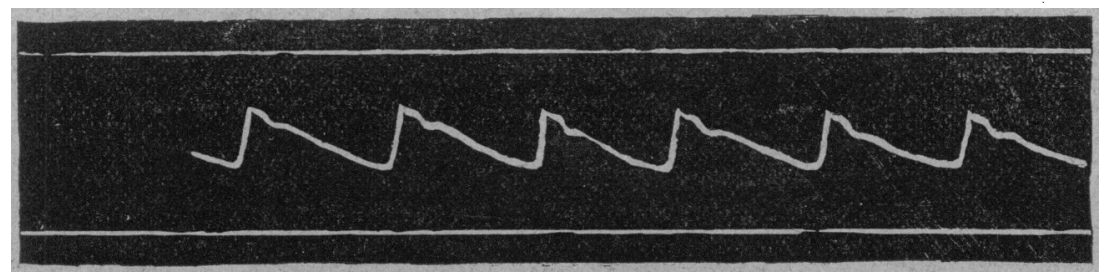

1. Williams (colored). Before bath No. 4, strength No. 1, Feb. 27, 1897.

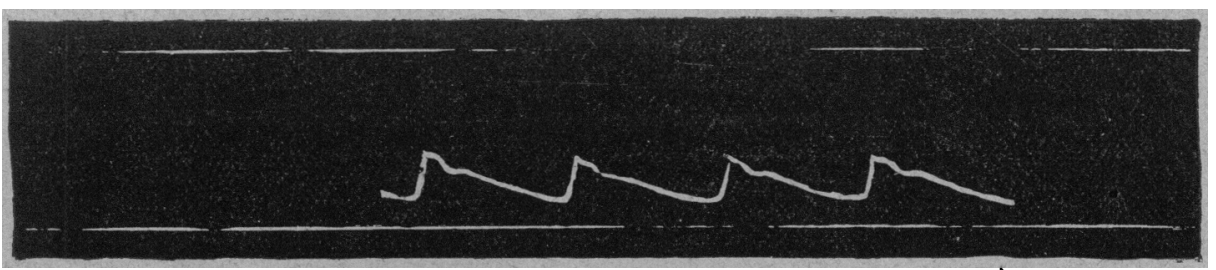

2. After bath.

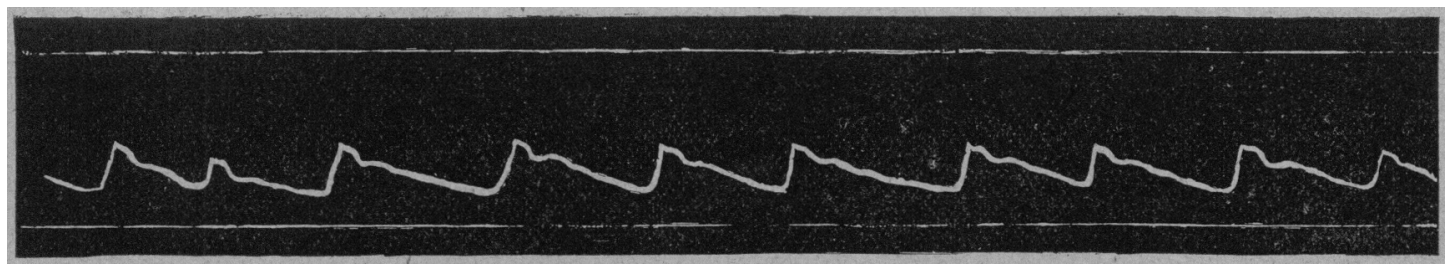

3. March 1. Before bath No. 5, strength No. 2.

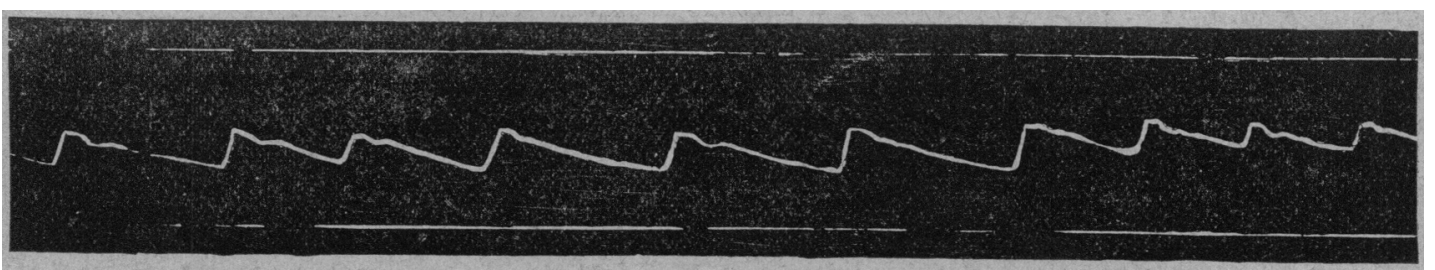

4. Immediately after bath.

Chart No. 7-Note. By some mistake strengths Nos. 1,2 and 3 were given in succession. Patient had received three baths before above tracings were taken; the character of the pulse was much improved in spite of this active treatment. Compare these tracings under bath treatment alone with Chart No. 8, tracings taken under tr. digitalis treatment alone. 
Chart No. 7 (tracings taken by Dr. Reuling) shows the results from the baths and exercises, though if not less striking but more lasting change under the bath treatment.

too grave may be controlled by the treatment.

The second case was that of mitral. insufficiency, beat and cardiac outline do not necessarily indicate who was given both the baths and movements. There permanent good results.

was no ehange in the point of maximum impulse or area of cardiac dulness. The patient was discharged in good condition.

3. Arterio-sclerosis may not wholly contraindicate

The third case was one of tachycardia. The influ- tioned that in the cases of nephritis, diluents were

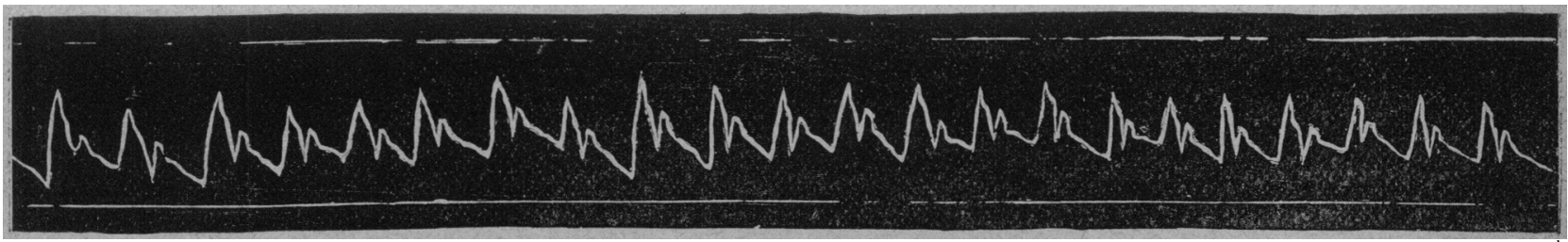

1. Before course of digitalis. Tr. digit. min. $\mathbf{x v}, \mathrm{q} .4$ hours ad. $3 \mathrm{ij}$.

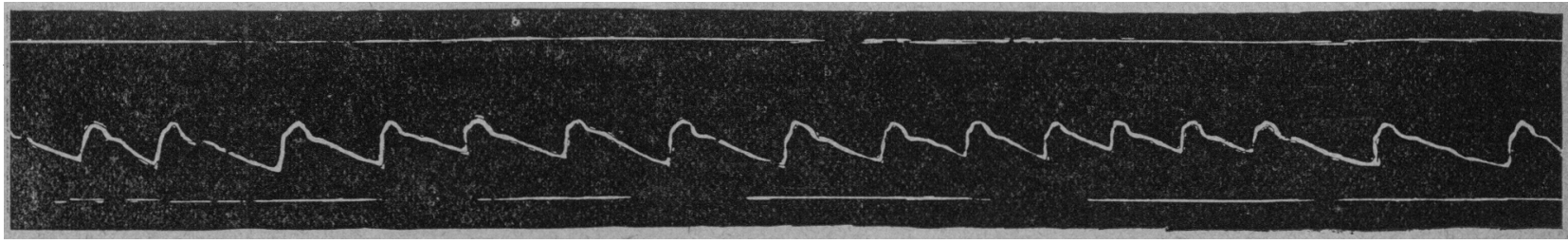

2. After course of digitalis.

Chart No. 8.- Hauser. Period of one day and eight hours. Compare witb Chart No. 7. where the period was three days under bath treatment alone. Slowing of pulse less marked with bath alone than with digitalis, but effects more lasting in former.

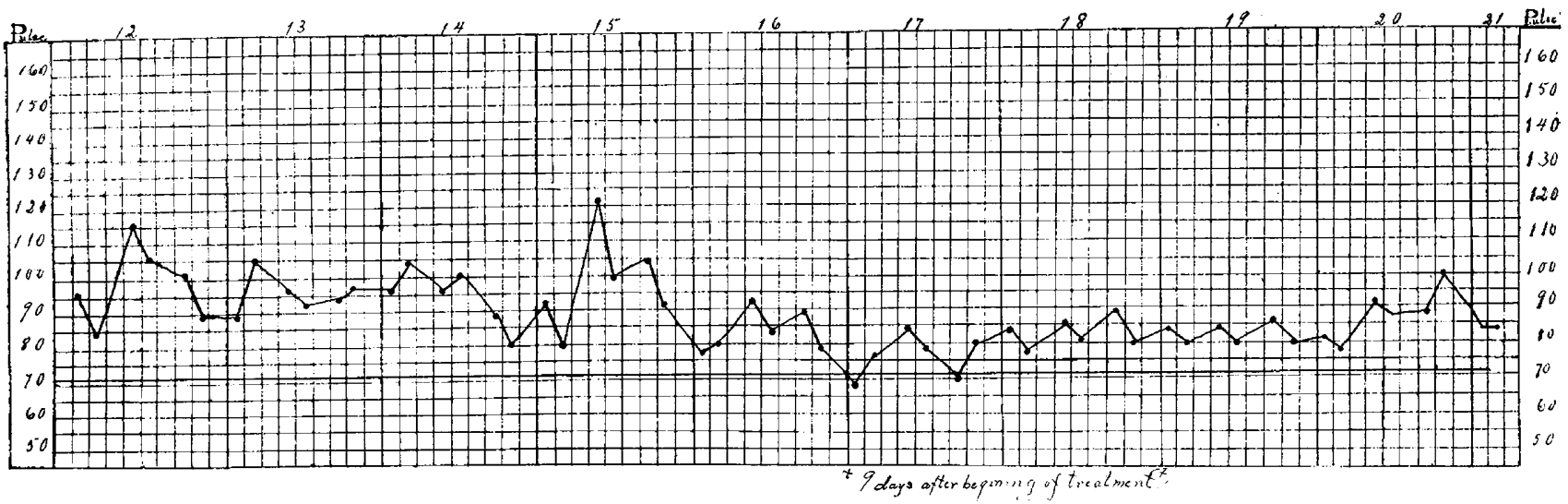

ChART No. 9.-Mrs. L. (tachycardia.) Resisted movements, group 1, Nos. 1, 2, 4, 5, were administered daily, patient in recumbent posture. Ghart showing steadying of pulse.

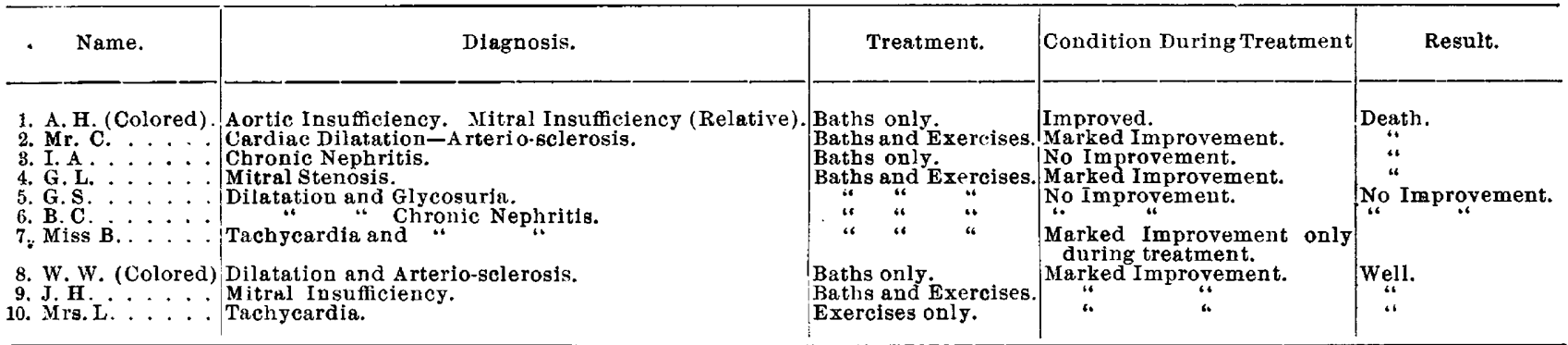

ence upon the pulse is seen in Chart No.9. Of these / freely given. Infusion of digitalis was employed in three cases of continued improvement it will be noted dram doses every four hours and occasionally the that very little change took place in the point of max- tincture of digitalis was used. Potassium iodid was imum impulse, whereas in those cases which showed administered, although sodium iodid is considered rapid improvement at first and which afterward ter- preferable. Strychnia, morphia, tr. strophan., nitrominated fatally, the change in apex was very striking. Iglycerin, were also used in some of the oases.

As far as our ten cases go the results are in favor of The diet laid down in our rules, while not strictly the following three points:

adhered to, was in the main followed out. While we

1. Extensive nephritis is a serious obstacle to good have found no consistent change in the point of max- 
imum impulse or in the cardiac outline we have found in every case a slowing and strengthening of the pulse and a diminished tension. We regret that owing to the inability to procure a suitable arteriometer that the caliber of the radial was not properly recorded. It is our hope to pursue systematically the effect of the baths and exercises upon the kidneys. There seems to be here an important field for the treatment.

Naturally as yet our experience is very limited and this report is made simply to indicate the mode in which the measures are being tested at the Johns Hopkins Hospital.

\section{DISCUSSION.}

Dr. WM. OsLER of Baltimore - The introduction of the Schott method into this country is due, I think, to Dr. Babcock of Chicago, who demonstrated that it may be efficiently carried out in hospital practice and in private work. I have had cases treated at Naubeim with rather extraordinary results, and I have long known of the great enthusiasm displayed by Theodore Schott in his advocacy of this treatment. I think it will be, in a certain group of cases, a very positive advantage. The method of carrying it out is a little irksome and troublesome, but in any well-managed hospital, and indeed in private prac tice, it is perfectly feasible.

Dr. J. F. Jenkins of Michigan-I would ask Dr. Osler in what class of cases this treatment is indicated.

Dr. OsLen-It is more particularly indicated in limiting the effect of cardiac dilatation without advanced nephritis and without much sclerosis of the arteries. Valvular disease itself is not a contra indication, and I think the most suitable group of cases are those of dilatation of the heart without much degeneration of the muscular substance, more particulariy cases occurring in comparatively youthful subjects, or in men between 40 and 45 years of age who have persistently eaten much and taken too much beer.

Dr. Charles Stockron of Buffalo-I would call attention to the fact that by the Schott brothers great stress is placed on the manner of conducting these movements so that the patient may not be overtaxed and more harm than good be done. A second point to which I would call attention is the carbonic acid in the artificial bath. I feel satisfied that the mixture recommended by Thorn is not of much use, for the gas escapes as rapidly as it is produced, and has little effect on the skin. To overcome this difficulty, $I$ have resorted to the use of an ordinary soda-water tank, and by conducting the escaping fluid under the water in the tub have been able to produce marked stimulation of the skin, and I feel convinced that this is a valuable addition to the saline constituents.

A third point relates to the mode of action of the bath upon the skin. I listened with much interest to what the reader said, and his explanation of this apparent stimulation of the capillary circulation, but I feel that there is still something wanting. The mere dilatation of the capillaries by heat, or by salt, or by any other means, does not necessarily increase heart action. Now, it seems to me there must be someting else in the action of this bath which adds certain force to the circulation. My friend Dr. Woods Hutchinson has an idea that there is communicated to the arteries a real propulsive force through the rhythmic contraction of the arterioles as a result of this stimulation.

Dr. Foster of Pennsylvania-I should like to know whether the author tried the ordinary bath in the treatment of any of these cases. He has related ten cases treated by the Schott method, and I should like to know whether he would have us infer that he regards that method with favor. Certainly the report of the cases would seem to be against it.

Dr. Herrick of Cleveland, Ohio--It seems to mo that this subject has been treated within somewhat narrow lines; that an organ is being considered independently of the organism, and stress is placed on the importance of special treatment by special means, withoutconsideration of the many circumstancesleading up to the condition. We understand that the heart is subject to diseases in itself, and it is subject to disturbances of function by reflex relation to other organs. I am not quite satisfied with the etiology as stated. The treatment, it seems to me, has no reference to the etiology of the disease. Therefore I regard it with suspicion. The idea of the application of baths for an organic or functional disease involving the heart is somewhat beyond my comprehension. There is an antecedent condition which we should look after that involves the nutrition of that organ. That the reflex influence on the heart of the propulsive force of the arteries, etc., should produce any prac tical results I am not able to see. I am not familiar with this Schott treatment, so called. I wish to see the rationale between the therapeutics used and the disease. It seems to me that any treatment adopted should have a relationship to the etiology of the organic condition. I go back in my investigation of this subject to the question of nutrition-the nutrition of the heart. The heart may be disturbed by a variety of influences, mental or nervous or nutritional. I would say go back and look into the digestive organs. All nutrition depends upon certain factors, and all diseases involve disturbances of nutrition. We know that the heart is in more intimate sympathy with the digestive organs than with any others. Therefore, the first attention, and constant attention, should be given the digestive organs. In this it seems to me that we have a practical, a rational line of treatment, somewhat more definite than that of baths.

Dr. SMITH of New York-The paper is pointed and practical. It is practical, inasmuch as the reader took into consideration the very facts referred to by the last speaker, those bearing on the relations of the general system to the disease of the heart. In the consideration of almost any disease we can not leave out the intimate relation existing between the different portions of the organism, and the sooner we $6 x$ in our minds the fact that it is the individual and not the disease that we have to treat, the better will be our results. There is a condition of sclerosis in which the general arterial system is involved in the first instance and a secondary effect is shown in the condi tion of the heart. I am very glad the first speaker (Dr. Osler) alluded to the effect of beer, or any kind of alcohol, but especially beer, in producing certain organic changes in the heart, what the Germans call the beer-heart. That is, it seems to have a transforming influence upon the capillary circulation, and that reacts upon the heart. Now, at the New York School of Clinical Medicine, I have used for the last six months the Ling system of physical exercise in the treatment of heart disease, and there is no question in my mind that that system when thoroughly carried out in appropriate cases has a marked influence in reducing the area of cardiac dulness. In the discussion which took place at the British Medical Association, by Thorn and Granger Stewart, due stress was laid upon the why and wherefore of the Schott exercises and due regard was given to the fact that it produced dilatation of the arteries throughout the muscular system, and consequently the propulsive power of the heart while not increased as to quantity was improved as to quality. That is, while the propulsive power of the heart was not increased in pulsation because it was slow, the heart muscle was toned up and intensified.

Dr. Jones of Buffalo-Schott has observed that the cardiac area has diminished and the pulse increased in volume from exercise alone and in a number of cases under baths. As I understand it, suitable cases after being treated there, are sent to the mountains, where the Örtel treatment may be car ried out and after time come back to Nauheim and are treated again. The compensation does not last longer, perhaps, than it would under due rest in bed and digitalis.

Dr. W. B. CAMAC-In reading up the reports on this treatment it is very difficult to approach it without a biased mind. We decided to try the treatment and see absolutely what it would do, and the report of the ten cases have been pre sented from an unbiased standpoint. I think we have not been misled one way or another at Johns Hopkins, and in reply to the remark about being for or against the treat ment, I may say that I believe we are neither one way or the other. We are still observing. I did not mean to slight the importance of the exercises, but they have been considered so frequently and in such detail that it did not seem recessary to weary you with the drill. This we have entrusted to the nurses. The importance of the very lightest resistance is made a great deal of, and one who is familiar with the treatment soon realizes how important it is to avoid all undue resistance which might lead to fatiguing of the patient. We have, therefore, trained nurses who administer the treatment under the supervision of a physician. The exercises are given as much attention to as the baths. We have found that carbonic acid gas can be satisfactorily formed according to the suggestion of Thorn, but not waiting five minutes, for in five minutes it has largely lost its power. Put the patiert in almost imme diately after the two ingredients are mixed, and the effect is accomplished in four or five minutes. If it is necessary to continue it, put in only one-half of the mixture at first and the other half at the end of five minutes. The temperature of the bath is 82,85 to 100 degrees $F$. The comparative effect of the ordinary hot bath and the Schott bath we have shown in the table. The baths were given in those instances alternately, the hot and the Scho't. In most cases the amount of urine is greater after the Schott bath than after the hot bath. We 
must be governed by what we find. There is absolutely nothing to be learned from mere theorizing, and I trust the facts presented in the paper and the diagrams will be the guide, not theory. They show that the salt bath has a greater influence on the kidney than the simple hot bath.

As to the etiology and the diet, we have laid down carefully studied rules which I think it is unnecessary to weary you with. The character of the diet, how much to give each day and the amount of wine or stimulants is carefully noted.

The Örtel treatment is carried out at Nauheim by sending the patients to walk up the Johannnesberg. As to the etiol ogy, it is impossible to go into that, because the amount of changes which may follow any influence upon the heart is legion. To attempt to associate the lesion with the treat ment is impossible. We can only say that the heart is dilated, its propulsive power is diminished. There is, there. fore, a back pressure; there is cyanosis, a congestion. If in any way we can dilate the peripheral vessels and allow more room for that blood which is pressed back into the system, we are perhaps going to relieve the heart of its overwork and improve its nutrition, so that the dilatation may end in hypertrophy and compensation.

\section{CLINICAL AND PATHOLOGIC FEATURES OF TWO CASES OF TYPHOID MENINGITIS.}

Read in abstract before the Section on Practice of Medicine, at the Forty-eighth Annual Meeting of the American Medical Association, at Philadelphia, Pa., June 1-4, 1897.

BY A. P. OHLMACHER, M.D.

DIRECTOR OF THE PATHOLOGIC LABORATORY OF THE OHIO FOSPITAL FOR EPILEPTICS, GALLIOPOLIS, OHIO; PROFESSOR OF PATHOLOGY IN THE CLEVELAND COLLEGE OF PHYSICIANS AND SURGEONS.

It will scarcely be necessary to point out, to an audience composed of clinicians and pathologists of large experience, the comparative rarity with which typhoid meningitis is encountered. In fact, the presence of an actual meningitis was so rarely demonstrated in typhoid fever that much doubt existed, only a few years ago, as to the occurrence of this lesion purely typhoidal in its origin. Since 1890, however, several cases of typhoid meningitis have been studied by careful bacteriologic and pathologic methods, so that there can no longer be a reasonable doubt concerning the identity of this complication of typhoid fever.

In a comparatively small number of autopsies on typhoid fever cases, it has fallen to my lot to meet with three instances in which a cerebral meningitis could be demonstrated, and in two of these cases a thorough bacteriologic analysis revealed only the bacillus of typhoid fever as the infecting micro-organism. These two cases were, therefore, looked upon as examples of true typhoid meningitis, and it is to a report on them that your attention is invited. The third case, in which a mixed streptococcus and typhoid bacillus infection was found, has just been reported.'

The source of the clinical data for these cases was the records of the Cleveland City Hospital.

Case 1.-Typhoid fever of four weeks duration. Marked delirium in last week preceding death. Autopsy shows usual typhoid lesions of fourth week with acute hemorrhagic internal cerebral pachymeningitis and exudative leptomeningitis. Typhoid bacilli isolated from spleen, mesenteric glands and from meninges.

J. K., Irish, laborer, 25 years old, single, admitted to the service of Dr. A. F. Spurney at the Cleveland City Hospital Jan. 18,1896 . His father and mother are both alive, and brothers and sisters alive and well. Aside from an attack of measles in childhood, patient does not recall any previous acute illness. He had a suppurating bubo in right groin nine years ago; no further venereal history. A month ago he went to work on an Ohio River boat, making a two weeks' trip which involved a great deal of hardship, and induced several attacks of diarrhea. Two weeks ago he went to Pittsburgh, stayed a week, then returned to Cleveland. Six days ago he again had

1 The Cleveland Medical Gazette, May, 1897. diarrhea, with a feeling of languor and aching in the legs. Diarrhea persisted till his admission to hospital. The temperature on entrance (afternoon) was 103 degrees F., and the chart shows a fairly typical typhoid curve during the life of the patient, though the fever was unusually high (botween 104 and 105 degrees F.) until death. The pulse averaged about 120 beats per minute. The urine was acid, contained a small though constant amount of albumin, and gave the diazo reaction. There were no other records to be obtained except that the patient was continually delirious during the last week of his life, and that he several times passed his urine involuntarily during this period. No suspicion of meningitis was entertained. Death occurred Feb. 3, 1896, seventeen days after entering the hospital.

Autopsy.-Fourteen hours after death. The body, which had been kept in a cool place, that of a man five feet, six and onehalf inches in length, and weighing about 125 pounds, showed no significant cutaneous markings. The pleural sacs contained no free fluid. Both lungs were moderately adherent posteriorly. The pericardium contained 90 c.c. of clear fluid, but no other evidences of inflammation. The heart was of normal size and in the normal location. There was no excess of fluid in the abdomen, and the abdominal organs were normally located.

Both lungs were of ordinary volume, with edematous upper lobes, and hypostatic congestion of the lower lobes. The pulmonary pleura, corresponding to the areas of adhesion, was thicker and more opaque than the remainder of this membrane. The bronchial glands were softened and swollen, while the bronchi showed no gross lesion.

The heart was flabby, the surface smooth, the myocardium pale and more than ordinarily friable. The endocardium was intact.

The spleen weighed 220 grams, was swollen, with tense smooth capsule. The pulp was soft and dark red. The kidneys were of normal size. The capsule stripped easily. The cortex was pale, and, relatively, somewhat increased in thickness. The surface of the liver was smooth, the organ pale, the boundaries of the lobules not well defined, and the substance friable. The gall bladder showed no inflammatory lesions, and the ducts were intact. The pancreas contained small deeply injected areas in its substance.

Intestines. - The ileum of the small intestine was almost exclusively the seat of characteristic lesions of its lymph follicles. The solitary glands were generally swollen, and many of them in various stages of ulceration or of healing. The swollen follicles were especially numerous near the cecum. In the upper portion of the ileum the ulcers of Peyer's patches were generally rough, their floors being covered with necrotic debris or with granulation tissue; while in the cecal portion the ulcers were sharply punched out, with clean floors and overhanging edges. Several exceptionally large ulcers appeared in the ileum, close to its cecal junction. No ulcers were found in the appendix. Numerous swollen follicles and a few typical typhoid ulcers averaging half a centimeter in diameter were found in the colon about the cecal region. The mesenteric and retroperitoneal lymph glands were uniformly swollen and softened.

Brain.--The skull cap and the external surface of the dura presented no unusual features. As the dura was carefully stripped back it was found to adhere to the pia unnaturally over the right side of the cerebrum, by means of soft, gelatinous, yellowish flakes, and delicate fibers. This adhesion began about the region of the right Rolandic fissure and increased as the removal of the dura advanced. The gelatinous flakes give place to clots of blood which increased in amount until, over the anterior portion of the occipital lobe, a thick, quite dense mass of clotted blood was found producing a depression, roughly circular in outline, about $4 \mathrm{~cm}$. in diameter, and $1 \mathrm{~cm}$. below the surrounding level at its deepest point. This depression persisted throughout the subsequent handling and hardening of the encephalon. The mass of blood, or hematoma, measured about 120 c.c. in volume. The left side of the large brain was quite free from adhesions or hemorrhage, and no other evidences of this kind were found, except about the base of the brain where a few delicate fibers, not ordinarily encountered, were found between the hard and soft meninges. There was a general edema of the pia mater, and over the right cerebrum this subpial fluid was distinctly cloudy. Under the mass of blood the contiguous pia inclosed soft yellowish flakes, and here the cerebral surface was distinctly reddened. The dural sinuses at the base of the skull contained soft, red, non-adher ent blood clots. No excess of fluid, and no inflammatory changes were apparent in the ventricular cavities. Aside from the area depressed by the hematoma, the brain substance presented no gross lesions. The spinal cord was not examined.

Histologic examination.-Portions of the various tissues, aside from the brain, were fixed in Carnoy's chloroform-acetic 